\title{
-NOTES-
}

\section{THE SOLUTION OF NATURAL FREQUENCY EQUATIONS BY RELAXATION METHODS*}

\section{BY J. L. B. COOPER (Birkbeck College, London)}

The application of Dr. R. V. Southwell's relaxation method ${ }^{1,2}$ to the solution of natural frequency equations for systems with a finite number of degrees of freedom is discussed in this article. The first object is to discuss the conditions under which the procedure converges. It is shown that two criteria can be given, such that if the one is satisfied, the procedure converges to the highest mode, and, if the other is satisfied, to the lowest. This is of more than purely theoretical interest: for it is shown that the possibility of finding the highest mode directly can be used to simplify considerably the process of finding further modes.

1. The criteria of convergence. The equations to be solved will be written in the form

$$
\sum_{s=1}^{n} a_{r s} x_{s}=p^{2} \sum_{s=1}^{n} b_{r s} x_{s}, \quad(r=1,2 \cdots n)
$$

where $a_{r}$ and $b_{r s}$ are constants, $\left|a_{r s}\right|$ and $\left|b_{r s}\right|$ are symmetrical and the values of $p^{2}$ for which a solution is possible must be determined. We write

$$
\begin{gathered}
A=A(x)=\sum_{r s} a_{r s} x_{r} x_{s}, \quad B=B(x)=\sum_{r s} b_{r s} x_{r} x_{s}, \\
A_{r}=\frac{\partial A}{\partial x_{r}}, \quad B_{r}=\frac{\partial B}{\partial x_{r}}, \quad \lambda=\frac{A}{B} .
\end{gathered}
$$

Both $A$ and $B$ are positive definite forms. In general, $\lambda$ denotes a variable function of the variables $x_{s}$, but the letter $\lambda$ with subscripts will be used to denote constant values corresponding to specified selections of the variables $x$.

Suppose that $x_{k}$ is changed to $x_{k}+\Delta x_{k}$. The change in $\lambda$ is then

$$
\Delta \lambda=\frac{A+\Delta A}{B+\Delta B}-\frac{A}{B}=\frac{B \Delta A-A \Delta B}{B(B+\Delta B)}
$$

Now,

$$
B \Delta A-A \Delta \dot{B}=B\left\{A_{k} \Delta x_{k}+a_{k k}\left(\Delta x_{k}\right)^{2}\right\}-A\left\{B_{k} \Delta x_{k}+b_{k k}\left(\Delta x_{k}\right)^{2}\right\}
$$

In the relaxation method, a first guess is made to select an initial set of values $x$, and the corresponding value of $\lambda$, say $\lambda_{1}$, is calculated. Then, in the basic application of the method, one of the $x_{k}$ is adjusted to make $A_{k}-\lambda_{1} B_{k}$ zero: the $x_{k}$ corresponding to the largest $A_{k}-\lambda_{1} B_{k}$ is chosen. (In theory this is the best possible single adjustment that may be made; in practice it is rarely used as more useful operations can always be performed, according to the skill of the computer, who should always be thinking as

*Received Aug. 29, 1947.

'Southwell, Relaxation Methods, Oxford University Press, 1940.

${ }^{2}$ Pellew and Southwell, Proc. Roy. Soc. (A) 175, 262-290 (1940). 
far ahead as possible and therefore using his judgement to modify each adjustment he performs so that it will ultimately have the best possible effect when taken in conjunction with the adjustments which follow). The process is repeated a few times; then the value of $\lambda$, corresponding to the values $x$ so obtained, is calculated and used as the new value of $\lambda_{1}$ in the next stage of the process. If the initial values $x$ are correctly chosen, the values of $\lambda_{1}$ found by these successive steps converge to the lowest value of $p^{2}$ for which the equations (1) have the solution. We shall now proceed to give a rigorous proof of this last statement, and also to show how the process can, just as easily, be made to converge to the highest mode.

The value of $\Delta x_{k}$, the change in $x_{k}$, is determined by the condition that $A_{k}-\lambda_{1} B_{k}$ vanishes when $x_{k}+\Delta x_{k}$ is substituted for $x_{k}$. Hence

$$
A_{k}-\lambda_{1} B_{k}+2\left(a_{k k}-\lambda_{1} b_{k k}\right) \Delta x_{k}=0 .
$$

On substituting this value of $\Delta x_{k}$ in (3), we get

$$
\begin{aligned}
B \Delta A-A \Delta B & =\left(\Delta x_{k}\right)^{2}\left\{\left(B a_{k k}-A b_{k k}\right)-\frac{2\left(B A_{k}-A B_{k}\right)\left(a_{k k}-\lambda_{1} b_{k k}\right)}{A_{k}-\lambda_{1} B_{k}}\right\} \\
& =-B\left(\Delta x_{k}\right)^{2}\left\{\left(a_{k k}-\lambda_{1} b_{k k}\right)+\left(\lambda-\lambda_{1}\right)\left[b_{k k}-\frac{2 B_{k}\left(a_{k k}-\lambda_{1} b_{k k}\right)}{A_{k}-\lambda_{1} B_{k}}\right]\right\}
\end{aligned}
$$

after a little algebra, using $A / B=\lambda$.

It is plain from (2) that the sign of $\Delta \lambda$ is the same as that of $B \Delta A-A \Delta B$. Now for the first step in the process, $\lambda=\lambda_{1}$, and hence from (5) it is obvious that

$$
\begin{gathered}
\Delta \lambda<0 \text { if } \lambda_{1}<a_{k k} / b_{k k}, \\
\Delta \lambda>0 \text { if } \lambda_{1}>a_{k k} / b_{k k} .
\end{gathered}
$$

This leads to the criteria for convergence of the relaxation method:

(i) in order that the process may converge to the lowest mode, the initial values $x$ should be chosen so that the initial value of $\lambda_{1}$ is less than $a_{k k} / b_{k k}$ for all $k$;

(ii) in order that the process may converge to the highest mode, the initial values $x$ should be chosen so that the initial value of $\lambda_{1}$ is larger than $a_{k k} / b_{k k}$ for all $k$.

It will be explained shortly how these choices of the initial values $x$ can be made.

If the choice (i) is made, the first step of the relaxation process, with $\lambda_{1}=\lambda$, leads to a new value $x$ with a smaller $\lambda$. If $\lambda_{1}$ is changed to this smaller $\lambda$ and the process repeated, it is plain that the value of $\lambda$ will decrease further. In practice it is not convenient to change $\lambda_{1}$ after each step in the process, and if this change is not made we cannot use (5) to argue directly that a further step will decrease $\lambda$ still further, for the $\lambda$ in (5) would be less than $\lambda_{1}$. The sign of the first term in the bracket in (5) will always be positive, but the sign of the term involving $\left(\lambda-\lambda_{1}\right)$ may be positive or negative. However, if $\lambda_{1}$ is near to the lowest proper value the value of $\left(\lambda-\lambda_{1}\right)$ will be small, since from Rayleigh's principle $\lambda$ must always be greater than the lowest proper value. Even if $\lambda_{1}$ is not near to the lowest proper value, $\left(\lambda-\lambda_{1}\right)$ will be small if the process is not carried on for too many steps with a fixed $\lambda_{1}$. In any case, no matter how many steps of changing an $x_{k}$ to reduce an $\left(A_{k}-\lambda_{1} B_{k}\right)$ to zero are carried out with a fixed $\lambda_{1}, \lambda$ will not increase above this $\lambda_{1}$; for (5) shows that as soon as $\lambda$ approaches $\lambda_{1}$ its value will begin to decrease. It is therefore plain that the process will lead to continually 
decreasing values of the $\lambda$ calculated after a series of steps. In practice the relaxation process. is continued with a fixed value $\lambda_{1}$, so long as the residuals $\left(A_{k}-\lambda_{1} B_{k}\right)$ as a whole can continue to be decreased. It should also be remarked that for any assumed $\lambda_{1}$, one solution is given by $x_{k}=0$ for all $k$; the computer must avoid approaching this solution by multiplying each $x_{k}$ by a factor from time to time so that the greatest of the $x_{k}$ is kept at some constant magnitude.

Similar remarks, with obvious modifications, apply if the choice (ii) is made. $\lambda_{1}$ then increases continually.

Since the $\lambda$ are bounded above by the highest and below by the lowest characteristic values of the equations, the $\lambda_{1}$ must tend to a limit. This limit must be a proper value of the system, for all the equations (1) are then satisfied. The proper value in question could conceivably be one other than the highest or the lowest, but if this were to occur in actual computation it could do so only as a result of rare good fortune, and by choosing a higher (or lower) value of $\lambda_{1}$ than the proper value thus found we could proceed to find the actual highest or lowest proper values.

2. Practical applications. We shall now show how a suitable set of values $x$ to serve as the first approximation in calculating the highest mode can be found. The method applies equally well, with an obvious change, to the lowest mode, but in most physical problems a good approximation to the lowest mode can be guessed intuitively. Let $r$ and $s$ be the values of $m$ for which $a_{m m} / b_{m m}$ takes on its highest values. The highest value of $\lambda$ possible with all $x_{m}$ zero except $x_{r}$ and $x_{s}$ is given by the larger root of

$$
\left|\begin{array}{cc}
a_{r r}-\lambda b_{r r} & a_{r s}-\lambda b_{r s} \\
a_{r s}-\lambda b_{r s} & a_{s s}-\lambda b_{s s}
\end{array}\right|=0,
$$

a quadratic which is easily solved. The corresponding values of $x_{r}$ and $x_{s}$ are given by

$$
\left(a_{r r}-\lambda_{2} b_{r r}\right) x_{r}+\left(a_{r s}-\lambda_{2} b_{r s}\right) x_{s}=0,
$$

where $\lambda_{2}$ denotes the larger root of the quadratic. These values of $x_{r}$ and $x_{s}$, with the other $x$ 's zero, should be used as the starting point for the relaxation process.

3. Estimation of frequencies intermediate between the highest and lowest. In the relaxation process described in the references below, it is necessary to find the lowest mode first, and then in finding the other modes to correct the values of the $x$ 's found in the successive steps so that they contınually satisfy

$$
\sum_{r s} a_{r s} x_{r}^{(1)} x_{s}=0,
$$

where $x_{r}^{(1)}$ denotes the value of $x_{r}$ in the lowest mode. The fact that the highest mode can be found directly saves some time in this process. We shall now show that after finding the highest mode we can alter the problem to a new one so as to make the next highest mode of the old problem the highest mode of the new problem. When this is done, the steps for finding the other modes can be carried out without corrections to satisfy (7).

Let $\lambda^{(i)}$ denote the $i$-th proper value, $x_{k}^{(i)}$ the corresponding mode values of $x_{k}$, then we have

$$
\sum_{\cdot} a_{r s} x_{s}^{(i)}=\lambda^{(i)} \sum_{\cdot} b_{r s} x_{s}^{(i)}
$$


From this it follows by a well-known argument that if $\lambda^{(i)} \neq \lambda^{(i)}$

$$
\sum_{r s} a_{r s} x_{r}^{(i)} x_{s}^{(j)}=\sum_{r s} b_{r s} x_{r}^{(i)} x_{s}^{(j)}=0,
$$

and even if two proper values are equal we can choose the modes corresponding to them so as fo make (9) true. We shall also suppose the modes normalised so that

$$
\sum_{r s} a_{r s} x_{r}^{(i)} x_{s}^{(i)}=1
$$

Now consider the matrix $A^{(i)}=\left|a_{r s}^{(i)}\right|$, where

$$
a_{r s}^{(i)}=\sum_{k} a_{r k} x_{k}^{(i)} \sum_{e} a_{s e} x_{e}^{(i)}
$$

We have

$$
\begin{aligned}
\sum_{s} a_{r s}^{(i)} x_{s}^{(j)} & =\sum_{k} a_{r k} x_{k}^{(i)} \sum_{e s} a_{s e} x_{s}^{(i)} x_{e}^{(i)} \\
& =\left\{\begin{array}{lll}
0 & \text { if } & i \neq j, \\
\sum_{k} a_{r k} x_{k}^{(i)} & \text { if } & i=j .
\end{array}\right.
\end{aligned}
$$

Hence, from (8),

$$
\sum_{s}\left(a_{r s}-a_{r s}^{(i)}\right) x_{s}^{(i)}=\left\{\begin{array}{lll}
0 & \text { if } & j \neq i, \\
\lambda^{(i)} \sum_{s} b_{r s} x_{s}^{(j)} & \text { if } & j=i .
\end{array}\right.
$$

After finding the highest mode and proper value, say $\lambda^{(n)}$, we can form the matrix

$$
a_{r s}^{1}=a_{r s}-a_{r s}^{(n)} \text {. }
$$

The highest mode of the original system of equations is annihilated by this matrix, but in the system of equations

$$
\sum_{s} a_{r s}^{1} x_{s}=\lambda \sum_{s} b_{r s} x_{s},
$$

all modes save the highest remain proper modes with their former proper values: while the former highest mode now corresponds to the proper value zero. On finding the highest mode of the system (13) by the method described above, we get the second highest mode of the old system. This new mode can then be eliminated in its turn and the mode below it found.

The procedure recommended, therefore, is to find the lowest and highest modes in the normal manner, and then to find the second highest, third highest modes, etc., in order, in the manner described.

It is clear from (12) that the matrix $\left|a_{r s}-\sum_{l=1}^{n} a_{r s}^{(i)}\right|$ annihilates all the modes; and since these modes are independent vectors and every vector can be expressed as a linear sum in terms of them, it must annihilate every vector and therefore must be the zero matrix. We have therefore

$$
a_{r s}=\sum_{i=1}^{n} a_{r a}^{(i)} .
$$


After $(n-1)$ modes have been found, and the reduction of the matrix $a_{r}$, carried out for each of them in the manner described, we must be left with the matrix

$$
a_{r s}-\sum_{i \neq i} a_{r s}^{(i)}=a_{r s}^{(i)},
$$

where $i$ is the number of the mode left to be found. In this matrix the terms of any row are proportional to $\sum_{s} a_{s e} x_{e}^{(i)}$ so the remaining $i$-th mode can be found by solving the set of linear equations

$$
\sum_{s} a_{r s} x_{s}=a_{1 r}^{(i)}
$$

The reductions can often be carried out with advantage in terms of the matrix $b_{r}$; this is done by normalizing the modes so that instead of satisfying (10) they satisfy

$$
\sum_{r s} b_{r s} y_{r}^{(i)} y_{s}^{(i)}=1 \text {, }
$$

and calculating $a_{r s}^{(i)}$ from the formula

$$
a_{r s}^{(i)}=\lambda^{(i)} \cdot \sum_{k} b_{r k} y_{k}^{(i)} \sum_{e} b_{s e} y_{e}^{(i)}
$$

which is easily deduced from (8). This is particularly convenient in the most usual type of problem in which $b_{r s}$ is a diagonal matrix - in mechanical problems, those in which the kinetic energy can be expressed as a sum of squares. If $\left|b_{r s}\right|=\left|m_{r} \delta_{r s}\right|$ the modes must be normalized by

$$
\sum_{r} m_{r}\left(y_{r}^{(i)}\right)^{2}=1
$$

and then

$$
a_{r s}^{(i)}=\lambda^{(i)} m_{r} m_{s} x_{r}^{(i)} x_{s}^{(i)} .
$$

After reductions corresponding to all but the $i$-th mode have been made on the matrix $a_{r s}$, the remaining matrix is. $a_{r s}^{(i)}$ from whose rows the $i$-th mode can be found immediately.

\section{A NORM CRITERION FOR NON-OSCILLATORY DIFFERENTIAL EQUATIONS*}

BY AUREL WINTNER (The Johns Hopkins University)

Let $f(t), x(t), \lambda(t), \cdots$ denote real-valued, continuous functions on an unspecified half-line, $t_{0} \leqq t<\infty$. If $\lambda(t)$ is positive on this half-line, put

$$
\lambda^{*}=\lambda^{*}(t)=\lambda(t) \int_{t}^{\infty}(d u) / \lambda^{2}(u),
$$

provided that the second factor on the right of (1) is a convergent integral. Under this proviso, a direct substitution of (1) shows that, if $\lambda(t)$ is a solution of the differential equation $D_{f}(\lambda)=0$, where 